\title{
A study on Risk Evaluation of Revenue Forecasting Accuracy in Tax Administration in Malawi; A Time Series Approach
}

\author{
Stella Binauli Nanthuru \\ School of Management \\ Wuhan University of Technology \\ Wuhan, Hubei Province, China. \\ e-mail: binaulistella@yahoo.co.uk
}

\author{
Guihua NIE \\ School of Economics \\ Wuhan University of Technology, \\ Wuhan, Hubei Province, China \\ e-mail: niegh@whut.edu.cn
}

\begin{abstract}
Nobody can accurately predict amount of sales revenue expected. There is need for accuracy to eliminate risk of revenue loss. Surveys have shown that accuracy is the most important criterion in selecting a forecasting strategy. Which criterion then provides the most accurate forecast? Human judgment and software have been used to address this issue but the question is how do we judge the accuracy? Is there revenue leakage due to over or under forecasting, resulting from lack of accuracy on the methods used? This paper evaluates accuracy of revenue forecasted over eleven years in a semi-autonomous Tax administration, a revenue collection body which collects taxes on behalf of government in Malawi. We investigate whether forecasting method which was used was the most accurate, unbiased and efficient, and whether better forecasts could have been used. We compare different Mean Absolute Deviation (MAD) and Mean Square Error(MSE) calculated from different time series methods using software CB predictor to establish the most accurate method for forecasting revenue collection for this organization. Actual data provided by Ministry of Finance in Malawi was used. The study shows that the forecasting method used by the organization was not most accurate than using the Last Value Forecasting Method which had the lowest MAD among the other forecasting time series method that were tested. The current method used by the organization had huge forecasting errors. The paper further recommends the use of Last Value Forecasting Method, and suggests other forecasting objectives the organization's may have other than accuracy.
\end{abstract}

Keywords-mean absolute deviation (MAD); mean square error (MSE) seasonal factor; forecasting error; risk management

\section{INTRODUCTION}

The future success of any business depends heavily on how savvy its management is in spotting trends and developing appropriate strategies, this is aided by use of forecasting techniques (Hillier F, M 2014). There is no exception in forecasting revenues any government is expected to collect. Just as weather forecasting is important in helping one to decide whether to carry an umbrella or not so is revenue forecasting. Taxes are a major source of revenue for government expenditure. Some objectives of taxation include; collection of tax revenue to fund public services, redistribution of wealth between the rich and poor, stabilization of the economy, mobilization of capital for economic growth. (Fuller, 1965; Luoga, 2000)
Revenue loss risk through forecasting errors is inevitable. The risk can be in form of over/under forecasting, which by the end of the day complicates the whole tax system by setting unrealistic tax target. However, these risks can be eliminated through accurately predicting revenues. How do we accurately forecast revenue? Are there any common measures of accuracy?

This study evaluates accuracy of revenue forecasts, by government over a period of 11 years. It compares the projected revenues with the actual revenues collected by tax administrators same period by observing the trend and forecasting errors. The study then evaluates the most accurate forecasting method for the tax administration by using the common measures of accuracy MAD (Mean Absolute Deviation) and MSE (Mean square Error) using time series approach. Three Time series revenue forecasting methods: Last Value, Averaging, and Moving Average over a number of 11 years have been used based on the data available by investigating whether these forecasts are accurate, unbiased and efficient. The computer software CB predictor, an excel template designed to perform statistical forecasting within a spreadsheet in MS Courseware was used

The paper discusses data collected, its analysis and methodology, the results and gives a conclusion

\section{Literature REVIEW}

Management of an organization holds a key to success of a business for they are custodian of the key strategy formulations (Hillier F, 2014). They have the experience to observe trends of a business and formulate appropriate strategies (Winklhofer et al 1996). This aids in developing the best forecasting technique and have the best ability to have the most accurate forecast as research has suggested that the level of forecasting capability is associated with the level of sales forecast performance (Donna et al 2007).

(Mc Carthy et al 2006) have argued that accuracy is key in forecasting. (Shukla 2000) stated that there are many factors that determine accuracy of forecasting. These include judgement (Sanders and Mandrodt, 1994, Winklhofer et al 1996) experience of forecaster, intuition and trend, even though (Elgers et all 1995) argued that when judgement is used in forecast, there is no accuracy and reliability. However, Mahmoud et al. (1988) argues that accuracy is the most important criterion in selecting a forecasting strategy. The use of computer software also aids in reducing 
judgement errors. For example, when you have calculated Mean Absolute Deviation (MAD) and Mean Square Error (MSE) accuracy issues can be minimized by measuring forecasting errors thereby identifying risk levels.

Most scholars have recommended the use of historical data to come up with trends and base their decision on accuracy of forecasting (Webby and O'Connor, 1996).

How then do errors come in? some studies have argued that forecasts should be used as directional as they encourage setting of low targets so that superior performance is observed (Leitch and Tanner, 1995) furthermore, some have argued that judgmental forecasting is biased and has insufficient data to base forecasting on, which results into most errors. But (Jenkins 2000) was of the view that forecasts parameters can be estimated from earlier periods to eliminate a substantial portion of the systematic error in current forecasts thus improving accuracy. This reduces unsystematic errors. In agreement to this, study done by (Donna et al 2007) found that organizational factors also matter in forecasting revenues, these include customer satisfaction, benchmarking the market, and organizational capabilities. These have direct impact on forecasting.

There are studies that have been carried out to find out accuracy of forecasting methods used in an organization or a business (Fildes 1991). Some have concluded that there is bias in the methods used (Makridakis W 1998, Edmundson et al. 1988). Limited studies have been done in tax administration, therefore it is in this regard that this paper evaluates accuracy of forecasting method used in a tax administration setup by comparing MAD and MSE of different Time series methods to measure forecasting accuracy and efficiency and recommends a better approach to avoid revenue leakages.

\section{ReSEARCh Methodology AND FindingS}

\section{A. Data Collection}

Secondary Data was used in this study collected from Malawi's Ministry of finance and from Malawi Revenue Authority collection reports. 11 years of revenues collected, including revenue forecasts that were set by government through ministry of finance. MRA collects taxes on behalf of government, established in 2000 through Act of parliament.

\section{B. An Analysis of Revenue Collection (July 2004 to June 2015)}

Below are graphs (fig. 1 and 2) showing an analysis of forecasted tax revenues versus collected tax revenues for a period of 11 years, from tax year July 2004 to June 2015 (fig 1 ), and their corresponding growth rates i.e. both forecast and actual revenues collected for each year (fig. 2)

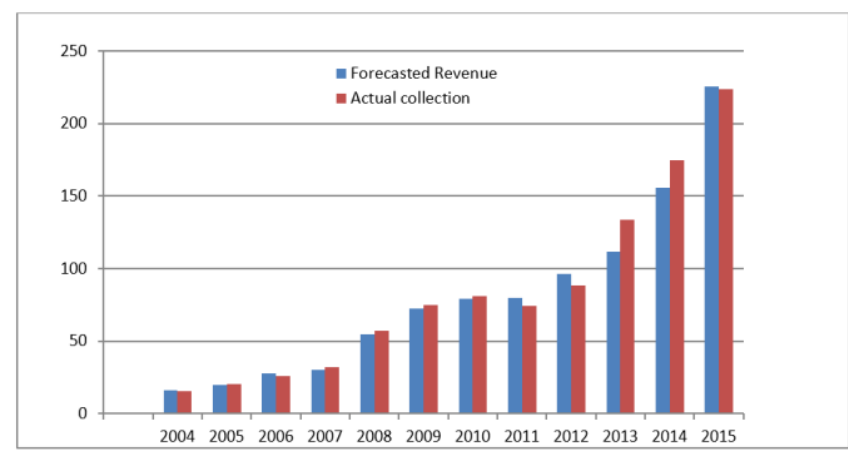

Figure 1. Actual revenue collected versus forecasted revenue for period July 2004 to june 2015. (Verticle axis is the money value in Billions of Malawi Kwacha)

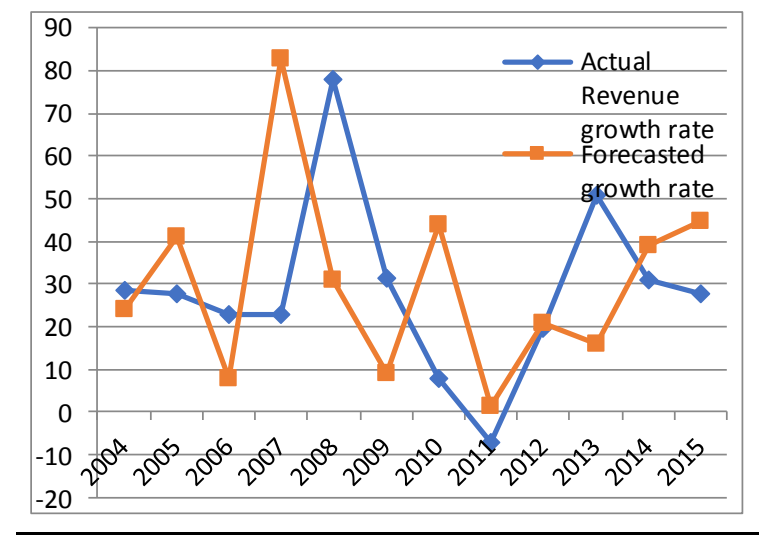

Figure 2. Actual revenue growth rate versus forecasted growth rate (Verticle axis is the \% growth)

We note from fig. 1 that revenue forecasted and revenue collected from taxpayers for the 11 years under review has a different trend pattern and this is evident in graph 2 where actual revenue growth rate compared to forecasted revenue growth rate do not correlate in many years. For example, in 2006 target growth rate was $8 \%$ while revenue grew by $22 \%$, likewise in 2008 target revenue grew by $31 \%$ while revenue collected grew by $78 \%$. The question is, could there be a problem with forecasting methods? Could there be inaccuracy in the forecasting method used? Could government be losing a lot of revenue by forecasting less or forecasting more thereby over collecting from taxpayers? These fluctuations and trends which are sharply shifting upwards and downwards clearly mean that the forecasting errors are huge. The trend for revenue growth rate and sales forecasted growth rate seems to sharply shift in the distribution of the growth pattern between the two variables which could mean error in forecasting (refer fig. 2)

To find out why, we take a look at the 3 qualitative Time series forecasting techniques then we forecast revenue based on the same data as above to evaluate correctness and accuracy of the forecasting method. We then calculated Mean Average Deviation (MAD) which is the average forecasting error to assess which method is the most accurate in terms of its deviance from the mean, the lesser the 
deviance, the more accurate is the forecast. We adopt these formulas for MAD and MSE:

Actual forecast $=$ seasonal factor $*$ seasonally adjusted forecast.

$\mathrm{MAD}=$ sum of the forecasting error/Number of forecast MSE = sum of square of forecasting errors/number of forecasts

We measure accuracy of the forecasting method by calculating Mean Square Error MSE) for each time series forecasting method used. MSE measure provides additional forecasting information about how consistently a forecasting method is to avoid serious errors.

\section{Last Value Forecasting Method}

Makridakis et al (1982) and Makridakis et al (1993) validated this method as a benchmark therefore we use this method to measure accuracy of revenue forecasting.

Fig. 3, shows what could have happened if this method had been applied to forecast MRA revenue true values for the period in question. It uses last true value to forecast future revenue.

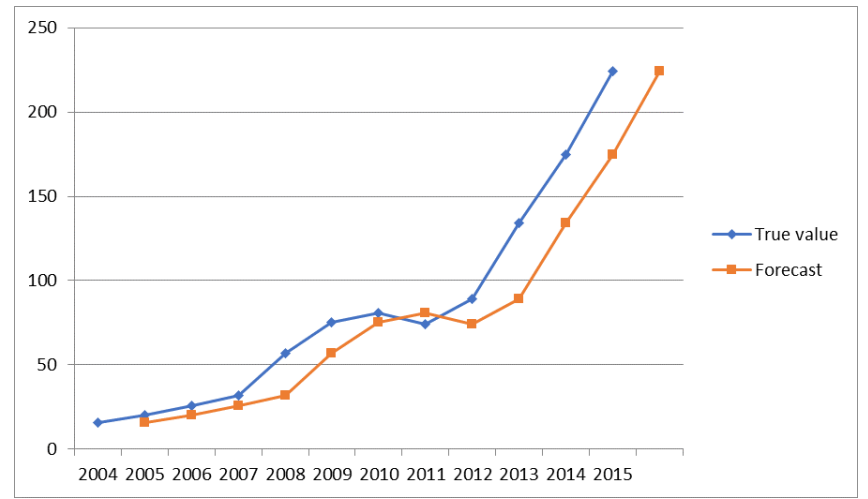

Figure 3. Revenue forecast using the last value forecasting method

The vertical axis represents monetary value (Malawi Kwacha in Billions), the $\mathrm{Y}$ axis represent time period.

An analysis of this method calculates MAD as 36 and MSE as 4802. In simpler terms, out of the 11 years of forecasting using this method, only 5 deviated with less than $10 \%$ from the mean, i.e. 2004, 2005, 2006, 2008, 2009. As for the rest, deviation varied, some up to $49 \%$. The more the deviation, the more the forecasting error.

The MES is calculated at 4802 which is very huge sum of money to forego because of forecasting errors.

\section{The Averaging Forecasting Method}

An average of all data in the sample is used rather than only using the last true value.

Below is a graph of forecast using true values, and using seasonal adjustment, then MAD and MES are calculated.

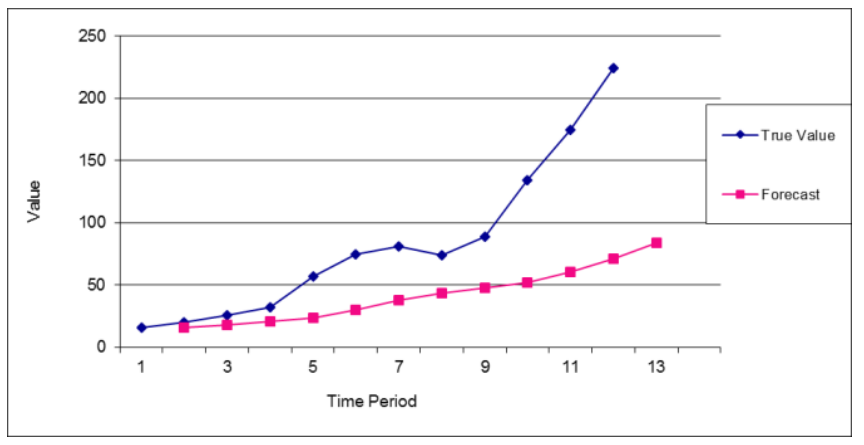

Figure 4. Revenue forecast using the average forecasting method

MAD calculated is 40, MSE calculated is 4,842

Time period 1 to 11 represents a time period of 11 years, 1 being 2004 and 11th being 2015

From the analysis, we note that at least 3 years (time periods 1 to 4) fall within the acceptable error margin which is at least $10 \%$, in 2005 forecasting error was at 5\%, $2006-$ $8 \%, 2007-10 \%$. The remaining years forecasting error is noticeably huge. The deviation from MAD would have been large hence questioning its accuracy.

MSE however is very minimal as compared to the former forecasting method, this might mean under forecasting

\section{E. Moving Average Forecasting Method}

This method averages the most recent data for only most recent time period $\mathrm{n}$ to come up with forecast. Which means forecast $=$ Average of last $\mathrm{n}$ periods

We assume our $\mathrm{n}$ to be 4 years. Below is the forecast

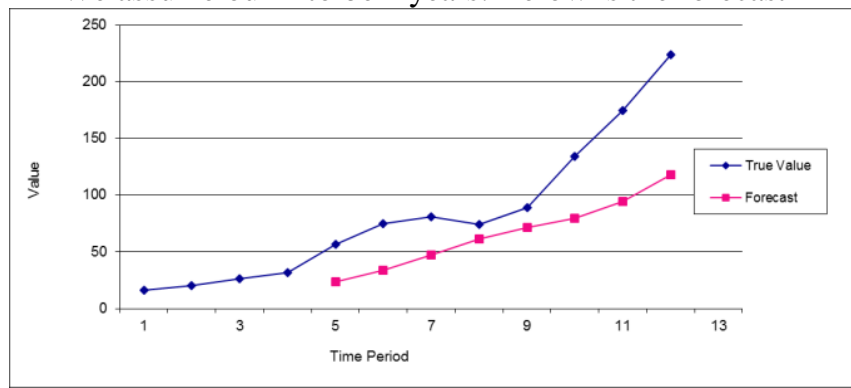

Figure 5. Revenue forecast using moving average method with seasonality

We calculate MAD as 47 and MSE as 3,133. This suggests that there could have been high chances of average error deviations from the standard mean in most years especially the later years from year 9 (2011) however the MSE is not as high $(3,133)$ as in the later method Averaging which had an MSE of 4,842.

\section{RESUlTS OF THE STUDY}

The following are the results of the study. From the data collected, risk levels have been calculated based on MAD and MSE to evaluate accuracy of the following forecasting methods used in the study; Last Value, Averaging forecasting and Moving Average. From figures 3, 4 and 5, the following findings can be summarized. Last value forecasting has a MAD of 36 and MSE of 4802. Averaging 
forecasting method has a MAD of 40 and MSE of 4842, and finally Moving Average Forecasting Method has a MAD of 47 and MSE of 3133. These results evaluate the forecasting errors of the 3 methods, Last value method having the lowest errors while Moving Average having the most forecasting errors. MSE results show that Moving Average has the least while Averaging forecasting has the largest MSE.

V. Discussion of Results, a COMPARATIVE ANAlysis of 3 Forecasting Time SERIES Method Discussed ABOVE USING MAD AND MSE

A summary of the Average Forecasting Error (MAD) and Mean Square error (MSE) for the various Time series forecasting method for MRA is summaries below:

TABLE 1 COMPARISON BETWEEN MAD AND MSE.

\begin{tabular}{|l|l|l|}
\hline $\begin{array}{l}\text { Forecasting } \\
\text { Method }\end{array}$ & MAD & MSE \\
\hline $\begin{array}{l}\text { Last Value } \\
\text { forecasting }\end{array}$ & 36 & 4802 \\
\hline $\begin{array}{l}\text { Average } \\
\text { forecasting }\end{array}$ & 40 & 4842 \\
\hline Moving Average & 47 & 3133 \\
\hline
\end{tabular}

Having compared the 3 methods above, it is noticeable that MAD (36) for Last value method is the lowest which translates into being more accurate than the other 2 methods, Averaging and Moving Averages. However, (Hillier 2004) argues that in using these forecasting techniques, we cannot conclusively say that the method with smallest MAD is more accurate than the one with highest MAD because some methods might have high MAD but lower MSE which translates into lesser risk in revenue loss even though it has the highest MAD. The moving average with highest MAD has a lowest MSE which translates into less risk in revenue loss, but on the other hand, it could mean that the target set were very low.

The averaging method however has a substantially highest MSE of 4842, this could probably be as a result of unstable economic conditions or increased tax rates. This method works better under stable conditions.

CONCLUSION

This paper evaluated accuracy of forecasting method used by tax administration in Malawi by comparing forecasted revenues with actual revenue collected for a period of 11 years from 2004 to 2015. To test accuracy of forecasting method used and to eliminate systematic bias in the forecasts (Goodwin, 1996), MAD and MSE were subsequently used on the three Time Series method, Moving average, Averaging method and Last Value. The results showed that forecasting revenue using the Last Value method is the most accurate as it gives a lower MAD than the other 2 methods. However, the moving average method gave the lowest MSE than the other methods which means that there is less revenue loss using moving average method than averaging method and last value.

Therefore, having serious fluctuations in revenue growth rate for both actual and forecasted revenues collected by the tax administration as evidenced in figure 2, this paper therefore serves as an eye opener for forecasters to reevaluate the revenue forecasting method used, and strike a balance between these methods to come up with a model that demonstrates the accuracy of the forecasting methods.

We can therefore conclude that, from the analysis and trend patterns observed in the study, last Value comes closer to reality in the current setup as it is a better reflection of what is projected and what has actually been collected unlike the pattern expressed in fig 2 .

The paper gives an emphasis on the importance of accuracy in revenue forecasting which is to eliminate revenue loss due to unforeseeable circumstances including economic changes. Again, another objective tax administration might consider, is using accurate forecasting as a tool to identify downtimes in the collection pattern and trend, thereby identifying risks and their corrective strategies, for continuous improvement through proper planning which is a backbone for every organization in its quest for improvement.

\section{REFERENCES}

[1] Mahmoud, E., Rice, G., Malhotra, N., 1988. Emerging issues in sales forecasting and decision support systems. Journal ofthe Academy of

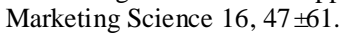

[2] Edmundson, R., Lawrence, M., O'Connor, M., 1988. The use of nontime series information in sales forecasting: A casestudy. Journal of Forecasting 7, 201 \pm 211 .

[3] Hillier,F.S, Hillier, M.S (2014), Introduction to Management scienceA Modelling and Case study Approachwith Spreadsheets.McGraw Hill Educationand China Machine Publications, 4th Edition. 380-415.

[4] Jenkins, G. P, Jenkins, Chun-Yan Kuo, Shukla G.P (2000), Tax Analysis and Revenue Forecasting. Harvard Institute for International development, Harvard University.

[5] Webby, R., O'Connor, M., 1996. Judgmental and statistical time series forecasting: A review of the literature. International, Journal of Forecasting $12,91-118$.

[6] Donna,F.,Davis,John.,2007. Organisational Factors in Sales Forecasting Management. International Journal of Forecasting Management 23 issue 3 p 475-495. 